\title{
Videoloop, una nueva forma de montaje cinematográfico y una aproximación metodológica para su enseñanza
}

\section{Videoloop, A New Way for Film Editing and a Methodological Approximation to Its Teaching}

\author{
Felipe Cardona (Colombia) \\ Pontificia Universidad Javeriana \\ Facultad de Comunicación y Lenguaje \\ http://felipecardona.blogspot.com \\ fluipe@gmail.com
}

\section{Resumen}

El lenguaje audiovisual, evidenciado en los tipos de montaje que describió Sergei M. Eisenstein en La forma del cine pareciera haberse detenido en el descubrimiento de sus recursos formales de imagen y sonido a finales de la década de 1920 con el Manifiesto del contrapunto sonoro. Lo que sucedió históricamente de la década de 1930

\begin{abstract}
Audiovisual language, evidenced in the types of editing described by Sergei $M$. Eisenstein in Film Form, seems to have stopped in the discovery of its formal resources of image and sound during the late 1920s with the Manifest of the sound counterpoint. What historically started in the 1930s was a stylistic exercise that responded to sociocultural tendencies.
\end{abstract}

\begin{tabular}{|c|c|c|}
\hline $\begin{array}{l}\text { FECHA DE RECEPCIÓN: } \\
\text { FECHA DE REVISIÓN: } \\
\text { APROBACIÓN: }\end{array}$ & $\begin{array}{r}26 \text { de noviembre de } 2013 \\
28 \text { de mayo de } 2014 \\
4 \text { de junio de } 2014\end{array}$ & $\begin{array}{l}\text { PARA CITAR ESTE ARTícULO / TO CITE THIS ARTICLE } \\
\text { Cardona, F. (2014). Videoloop, una nueva forma } \\
\text { de montaje cinematográfico y una aproximación } \\
\text { metodológica para su enseñanza. Poliantea, 10(18), } \\
\text { pp. 35-61. }\end{array}$ \\
\hline
\end{tabular}


en adelante fue más un ejercicio estilístico que respondía a tendencias socioculturales de cada época, como el neorrealismo italiano, el free cinema inglés, el cine independiente estadounidense o el dogma 95 (todos realismos) con algunos aportes formales al lenguaje, como la cámara al hombro, el sonido directo, el uso de actores naturales, la iluminación natural o el plano secuencia, pero más de orden ideológico que formal. Viendo la aparición de videos con una nueva y auténtica forma, como los publicados por Lasse Gjertsen o Bond TV, ambos en Youtube, hay que revisar si ciertamente corresponden a una nueva forma de narrar y usar los elementos citados por Eisenstein, o si es una revisión actual de un concepto ya citado, en este caso del de montaje rítmico, o si gracias a la tecnología de producción audiovisual del siglo XXI algunas ideas de los cineastas rusos de la década de 1920 se materializaron con elementos sonoros, concretando y transformando hoy un sueño del pasado.

Palabras clave: videoloop, montaje, Eisenstein, Youtube, cine.
That is the case of the Italian Neorealism, the British Free Cinema, the American Independent Film, or the Dogme 95, each of them belonging to realism, with some formal contribution to the language -such as over-the-shoulder shots, direct sound, use of natural actors, natural lighting, or sequence shots-, but more in the ideological than in the formal realm. Videos with new and authentic forms, as the ones published on YouTube by Lasse Gjertsen or Bond TV, make us wonder if they belong to a new way to narrate and use the elements mentioned by Eisenstein; if they are only a current version of a concept that was already mentioned /which is the case of the rhythmic editingl; or if thanks to the audiovisual production technology of this century, some ideas of the Russian film directors of the 1920s were brought to life with sound elements, thus setting and transforming a dream of the past.

Keywords: videoloop, editing, Eisenstein, YouTube, film. 


\title{
Videoloop, una nueva forma de montaje cinematográfico y una aproximación metodológica para su enseñanza
}

\section{Videoloop, A New Way for Film Editing and a Methodological Approximation to Its Teaching}

\author{
Felipe Cardona (Colombia) \\ Pontificia Universidad Javeriana \\ Facultad de Comunicación y Lenguaje \\ http://felipecardona.blogspot.com \\ fluipe@gmail.com
}

\section{Hipótesis}

Los tipos de montaje ruso y estadounidense señalados por Sergei M. Eisenstein en La forma del cine señalaban las posibilidades narrativas del cine antes de la aparición del cine sonoro. Cuando aparece el sonido y los cineastas rusos escriben el Manifiesto del contrapunto sonoro, trataron con este documento de darle un lugar importante al sonido dentro de los elementos del lenguaje audiovisual, y no relegarlo a complemento de la imagen. El montaje métrico y rítmico, conceptos del cine mudo, en unión con los conceptos derivados de la aparición del cine sonoro, han dado como resultado concreto un nuevo tipo de montaje, más de la mano de la música que de la literatura (si citamos a Edwin Porter o David W. Griffith y sus tipos de montaje estadounidense de inicios del siglo XX), que desde este artículo se le quiere 
llamar videoloop. En el videoloop, la reiteración en el montaje crea una ilusión de sentido.

\section{Introducción}

Ciertamente, el uso de reiteraciones para crear la ilusión de ritmo, o la exploración del ruido y voces para encontrar tono, no comienza en la década de 1980, ni mucho menos con la reciente mulitimedialidad de internet. A principios del siglo XX, durante sus primeras tres décadas, los cineastas que descubrieron los prodigios en la pantalla de Georges Méliès tomaron ese gran descubrimiento que fue el corte, y exploraron todas sus posibilidades, tanto narrativas desde una perspectiva literaria, como estéticas o de gran divertimento. Los cineastas estadounidenses, encabezados por Edwin Porter, luego de intentar imitar a Méliès y a los hermanos Lumière, decidieron darle al corte un uso netamente narrativo. Los rusos hicieron lo propio clasificando el montaje de acuerdo con duraciones y conceptos musicales. Los rumbos que el cine mainstream tomaron fueron más literarios que los experimentales y cercanos a la música que tomaron los cineastas rusos de la década de 1920. Con la llegada de Youtube y la democratización audiovisual, la experimentación y búsqueda por la musicalidad está en manos de más personas.

\section{Un lenguaje en construcción de menos de dos siglos de edad: la historia del cine como creación del lenguaje audiovisual}

Luego de los heroicos descubrimientos de Muybridge, de los emprendimientos ingeniosos de Edison y de la asertividad de los hermanos Lumière, fue Georges Méliès quien le dio destino y derrotero al descubrimiento que tal vez le diera forma a ese incipiente lenguaje cinematográfico a finales del siglo XIX: el corte. Se lee en reseñas varias, unas con tono histórico, otras con sabor a mito, que Méliès descubrió por accidente la interrupción y retoma de una acción mientras filmaba gente y movimiento, al mejor estilo de los Lumière, con su teatroscopio. A partir de ese momento, Méliès se toma la atribución de deformar espacio y tiempo en sus filmes y, partiendo del corte, se inventa técnicas de trucaje que serían definitivas para que cineastas del siglo $\mathrm{XX}$, al intentar imitarlo, descubrieran otras fronteras de ese naciente montaje. Mientras los Lumière y Edison se transaban en amargas e inútiles disputas legales, Georges no paraba 
de crear cortometrajes fantásticos. Ejemplo del fervor de este pionero a finales del XIX fue Un homme de têtes, cortometraje de 1898 en el que el propio Méliès se quita la cabeza y se multiplica a sí mismo por cuatro, a modo de acto de variedades.

Méliès quería con el cine y con el corte explorar lo imposible, lo ilusorio, era un creativo demoledor que a su manera y en su época quiso llegar a los límites de la forma, su interés era saber y dominar una artesanía que en la pantalla permitía la alteración de espacio y tiempo, que ofrecía una ventana de apariencia realista a un mundo imaginario. Otros realizadores, como Edwin Porter, capitalizarían el ingenio devastador de Méliès para darle un uso al corte que calaría más hondo en las audiencias primitivas de la sala oscura.

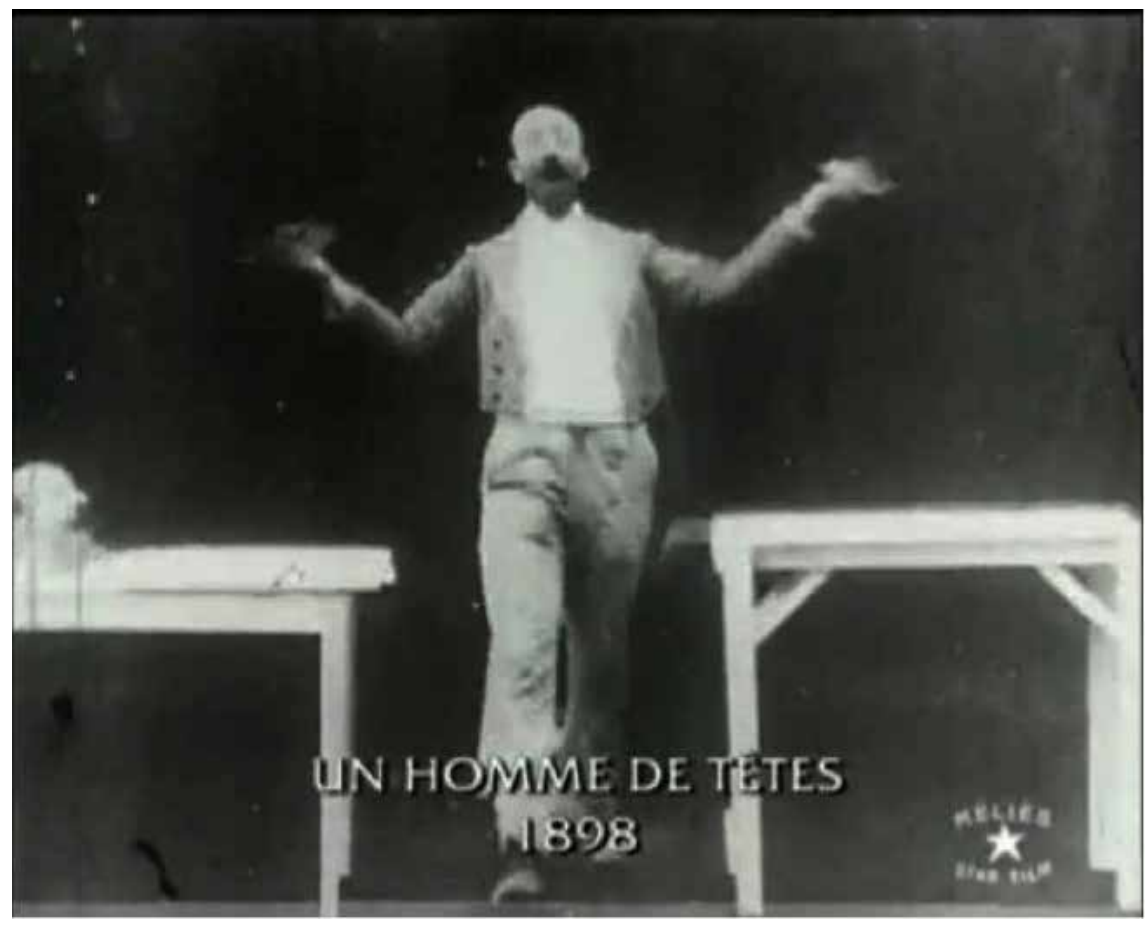

George Méliès, Un homme de têtes, 1898 (http://www.youtube.com/watch?̣v=8oFnOAnL8Ss) 


\section{Cine y literatura: ¿parentesco forzado o conveniente? \\ La cercanía del cine estadounidense a la literatura según Edwin Porter}

Fue Edwin Porter, iniciando el siglo $\mathrm{XX}$, quien comenzó su propia exploración de la forma cinematográfica, haciendo uso del corte e incluso imitando a Méliès. En sus primeros intentos con el cine, buscaba la novedad y la sorpresa en narraciones muy cortas, como What happened on Twenty-third Street, New York City, antecedente de 1901 de las "cámaras escondidas", con cierto aire documental.

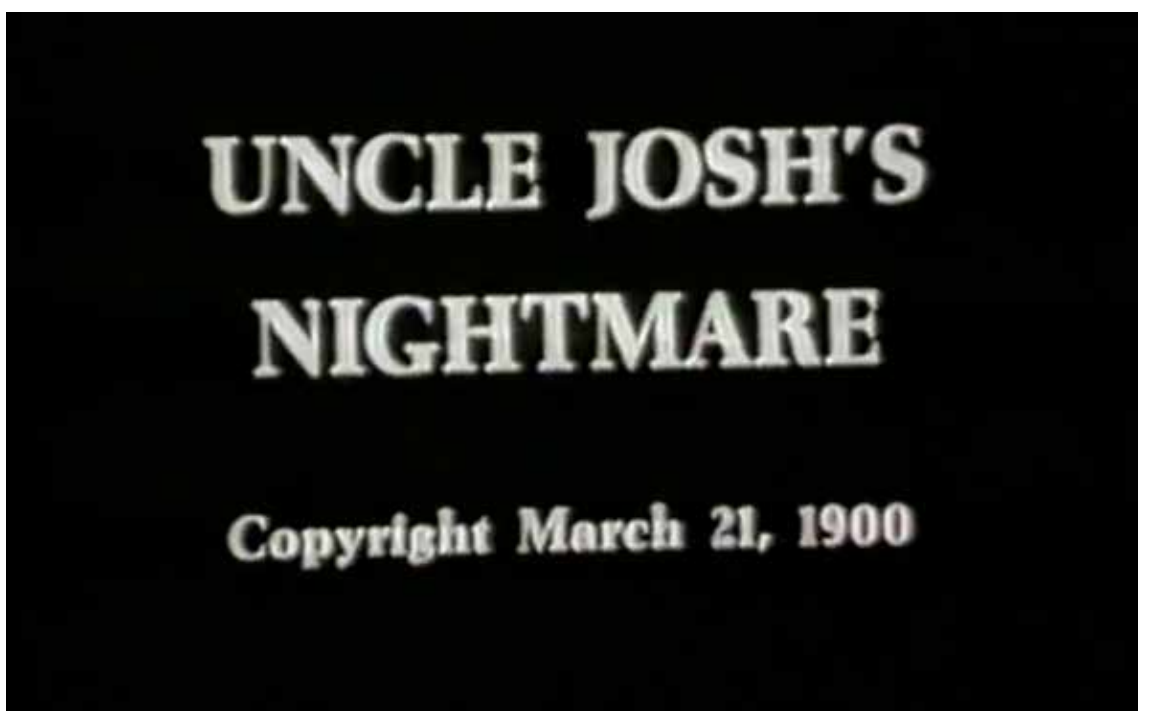

Edwin Stanton Porter, Uncle Josh's Nightmare, 1900 (http://www.youtube.com/watch?2v=HI_B38R)4zal). 


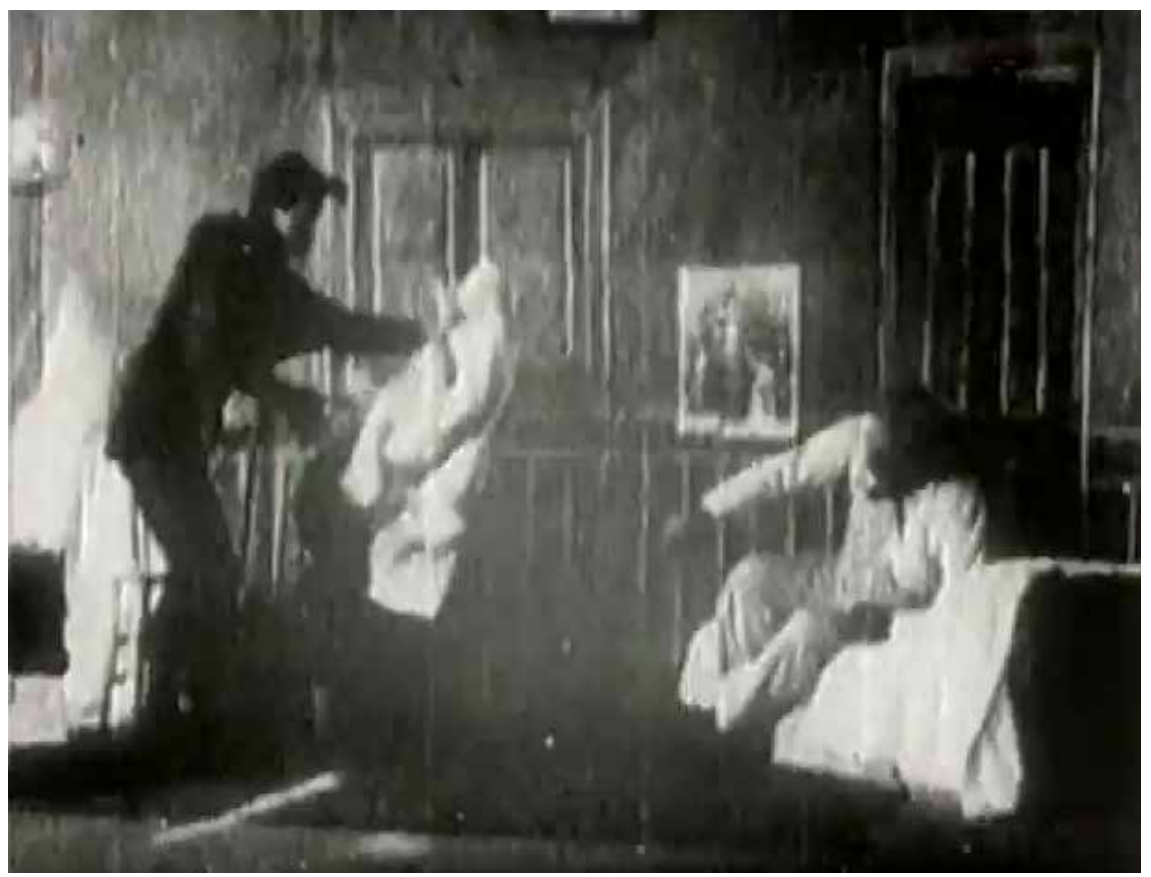

Edwin Stanton Porter, Uncle Josh's Nightmare, 1900 (http://www.youtube.com/watch??v=HI_B38R4zall).

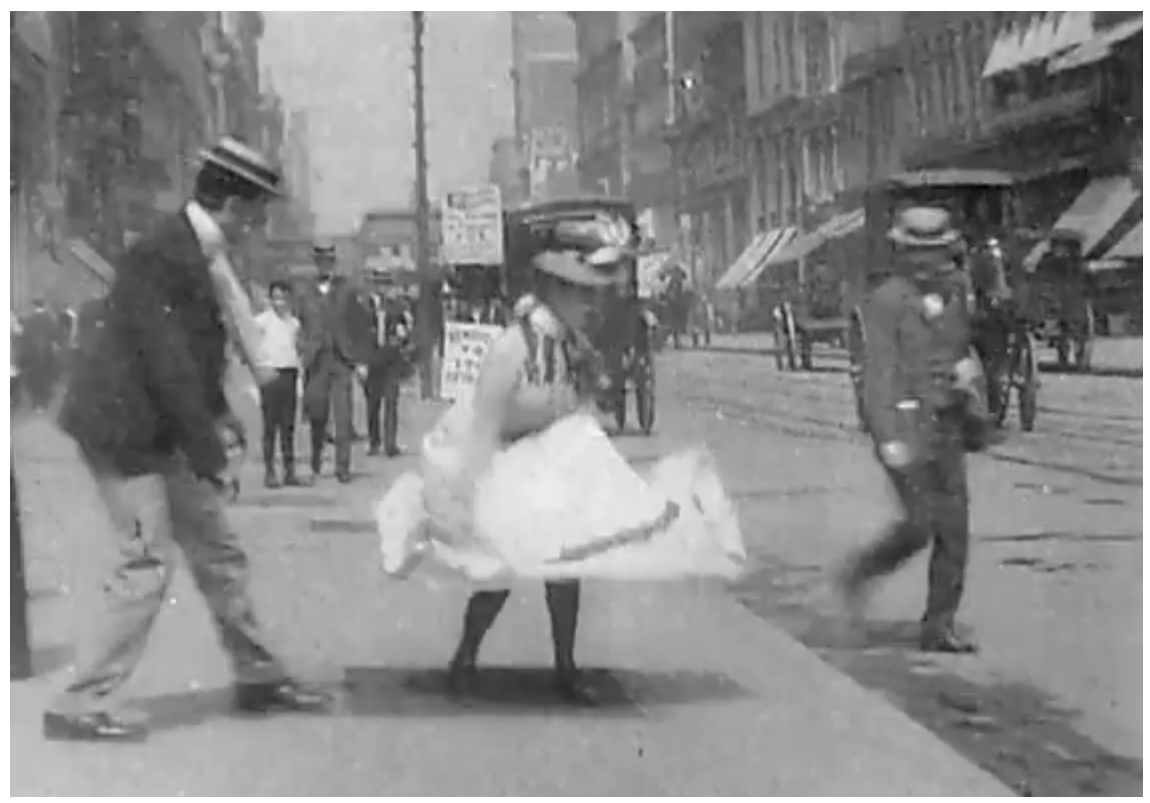

Edwin Stanton Porter, What Happened On Twenty-Third Street, New York City, 1901 (http://www.youtube. $\mathrm{com} /$ watch? $\mathrm{v}=\mathrm{C} 8 \mathrm{MbmaSOspU})$. 
En 1900 Porter hace Uncle Josh's Nightmare, cortometraje que se parece bastante a Un homme de têtes, de Méliès, en cuanto a que a través del corte, a la interrupción y retoma de una acción continua tomada con la cámara inmóvil, logra que sus personajes aparezcan y desaparezcan en un escenario que no varía.

No fue sino hasta 1903 que Porter hizo dos cortometrajes que marcarían en adelante el destino y la forma del cine estadounidense, mostrándoles a cineastas precedentes, como $\mathrm{Da}$ vid W. Griffith, la finalidad del cine: contar una historia y hacer negocio con su audiencia. En Life of an American Fireman y The Great Train Robbery, Porter usa el corte para hacer avanzar una historia de principio a fin de manera lineal, en la que el instante exhibido precedía en tiempo y espacio al siguiente, $\mathrm{y}$ así hasta culminar.

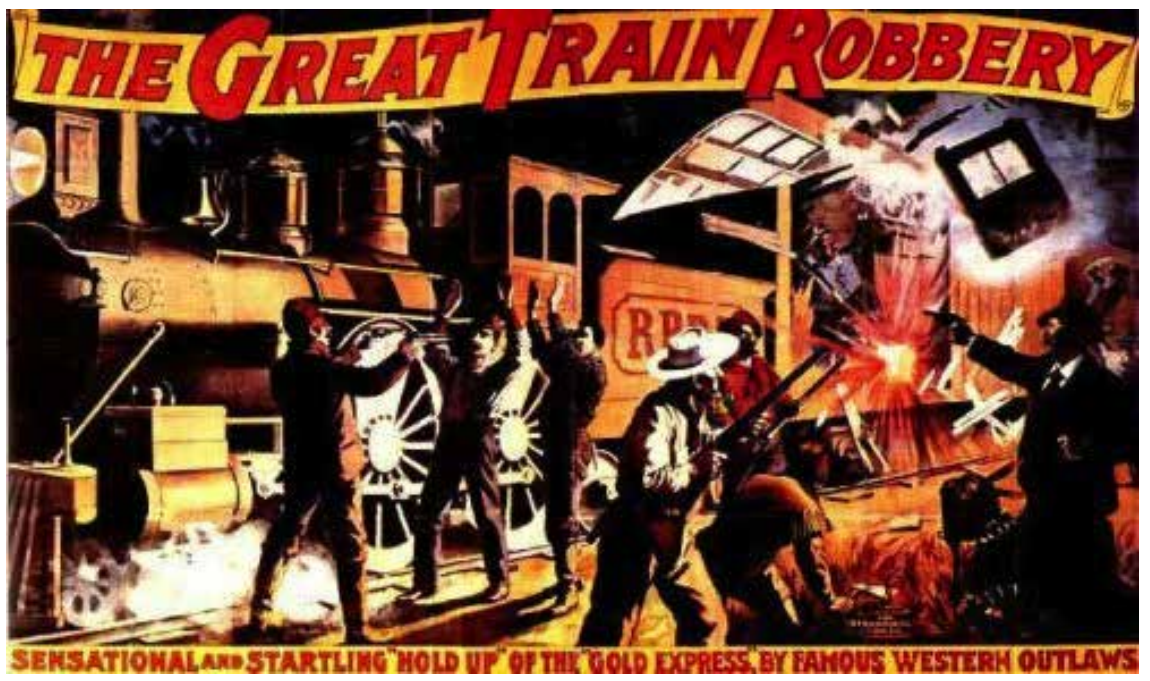

Edwin Porter, The Great Train Robbery, 1903 (http://www.youtube.com/watch?v=Bc7wWOmEGGY). 


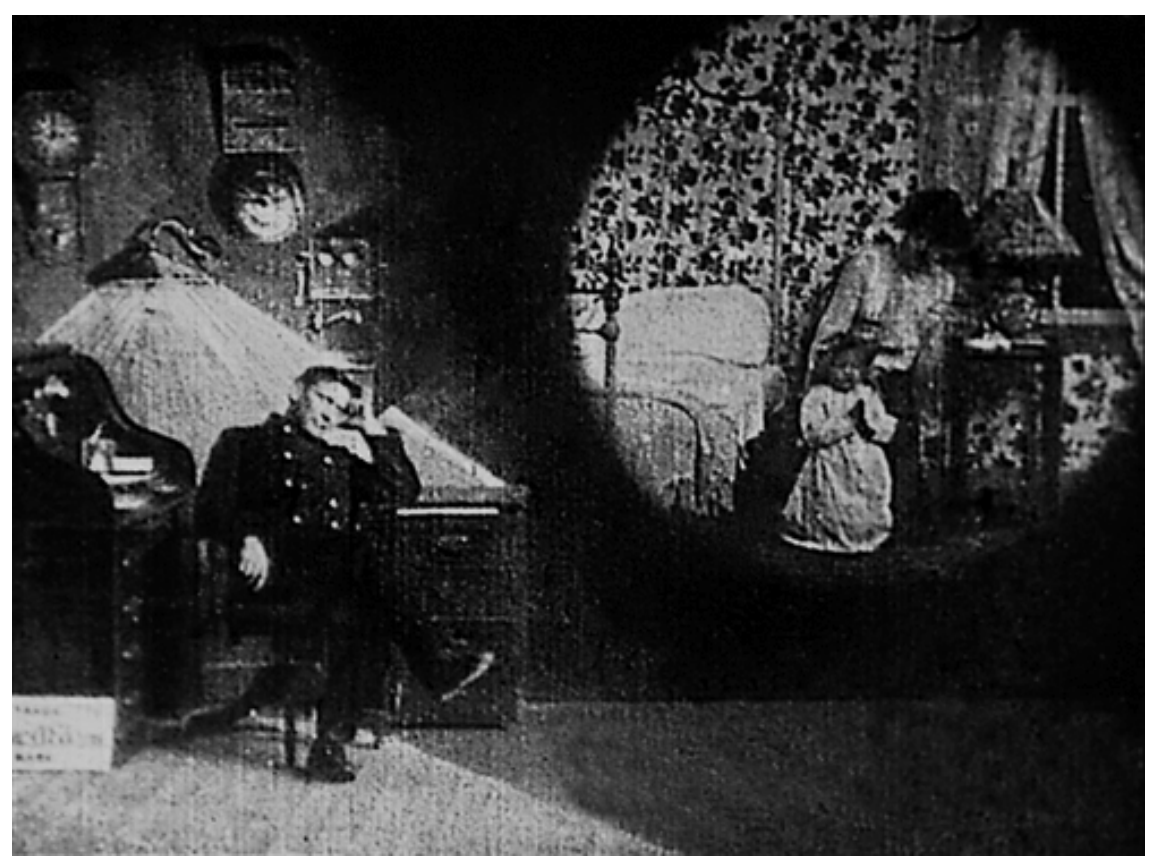

Edwin Porter, Life of an American Fireman, 1903 (http://www.youtube.com/watch?2v=p4COgJ7BnLc).

\section{Montaje: la esencia de la narración audiovisual. Clasificación para su entendimiento}

En Life of an American Fireman, Porter cuenta la historia de cómo unos bomberos apagan un incendio, y al inicio creó una de las imágenes más memorables del nacimiento del relato cinematográfico cuando se sugiere con una imagen en círculo al lado de un bombero dormido que el personaje intuye, sueña o simplemente piensa. En The Great Train Robbery, Porter apuesta por la misma linealidad, una narración de principio a fin en la que unos bandidos roban una estación de tren, luego un tren en movimiento y finalmente son abatidos. Sergei M. Eisenstein (1986), en su segunda colección de ensayos llamada La forma del cine, conceptualiza los estilos narrativos desde el montaje que se manejaron en esos primeros días del siglo XX en Norteamérica y Rusia, y los clasificó según el recurso narrativo de la intención. En muchas escuelas de comunicación o de cine, 
se habla de los tipos de montaje estadounidense, cuatro dentro de un grupo grande conocido como narrativo, y los tipos de montaje ruso, los de "la forma del cine", que obedecen a criterios de duración. Los cuatro estadounidenses, reconocibles sin dificultad en una película, son:

- Montaje lineal, cuando una acción ocurre después de la otra, hasta el final.

- Montaje paralelo, cuando dos líneas narrativas se alternan en la pantalla por momentos.

- Montaje invertido (flashback, flash forward), aquel que no se ciñe a la linealidad del tiempo, en el que se sugiere un sueño, recuerdo o anhelo.

- Montaje alterno, cuando se alternan dos líneas narrativas que, a su vez, cada una es un montaje paralelo.

Cuando Eisenstein compara a David W. Griffith con Charles Dickens en La forma del cine, quiere hacer notar el influjo de la novela victoriana anglosajona en el consecuente cine de principios del siglo $\mathrm{XX}, \mathrm{y}$ su notoria tendencia a la narración literaria traspuesta al montaje para la pantalla. Y ciertamente no es sino observar The Great Train Robbery y compararlo con, por ejemplo, A sangre fría, de Truman Capote, para reconocer de inmediato el montaje paralelo. La clasificación que sugiere Eisenstein en los métodos de montaje de La forma del cine obedece a otro criterio: se encuentra en terrenos de lo emotivo, y su clasificación hace referencia no a qué línea narrativa se muestra o cómo se mezcla con otra, sino a la duración de los planos. Eisenstein dice que hay:

- Un montaje métrico, cuyo criterio de ordenación es la longitud absoluta de los fragmentos, correspondiendo a un compás musical.

- Un montaje rítmico, en el que el contenido interno de cada plano influye en el ritmo que se le quiera dar, haciendo incluso que las duraciones reales de cada fragmento dejen de ser precisas.

- Un montaje tonal, en el que la duración de los fragmentos se supedita al tono de la escena o conjunto de trozos que se esté cortando, imponiendo un criterio superior al que pueda darse entre un plano al otro.

- Un montaje sobretonal o armónico, cuando hace referencia al 
tono de toda la obra y los choques que cada escena o conjunto de fragmentos plantea, siendo una búsqueda de la armonía.

- Un montaje intelectual, cuando superficialmente una escena está cortada con alguno de los métodos anteriores, pero por un contexto dado en la diégesis de la película, el espectador intuye un proceso interior del personaje que ve en la pantalla, algo similar al concepto de subtexto del teatro cuando se habla de algo irrelevante y la intención real la percibe el espectador aunque no se mencione.

Las clasificaciones de los tipos de montaje o formas de edición pasan por todo tipo de consideraciones, provenientes de lo histórico, lo formal y lo social. En Portalcomunicación. com de la Universidad Autónoma de Barcelona, hay un valioso intento por agrupar las múltiples y extensas teorías del cine. Fernández (2008), muy acertadamente, agrupa las teorías según el modelo básico de comunicación, en teorías de cine según la enunciación, el canal o el mensaje y la recepción. De acuerdo con Fernández, las teorías de emisión y recepción son ciertamente personalistas, en cuanto analizan el cine basadas en quiénes lo hacen y quiénes lo reciben, como en las teorías realistas de la posguerra de Bazin y Kracauer: la del cine de autor y la del cine del Tercer Mundo, agrupadas en la categoría de teorías según la enunciación, y también teorías, como la multiculturalista, feminista y cinéfila, agrupadas en la categoría de la recepción, afines al visionado y a la reacción de un público. Las teorías agrupadas en la categoría de mensaje o canal son ciertamente formativistas o formalistas, $y$ tienen la intención de evidenciar el proceso cinematográfico desde el ejercicio del montaje: pretenden exhibir el cómo con la intención de "dotar al análisis de un estatuto científico" (Fernández, 2008, p. 6). Tomando en cuenta que el ejercicio teórico derivado del experimento de Lev Kuleshov puede redundar en una interpretación mecanicista del cine, las teorías formalistas ciertamente dan bases tangibles para crear cine desde el entendimiento de la yuxtaposición. Son teorías para aspirantes a cineasta, no tanto para estudiosos de fenómenos sociológicos a causa del cine.

Es claro que entender y reconocer los tipos de montaje del cine estadounidense es más fácil que los del cine ruso. Los primeros se acercan 
a formas de leer novela o cuento, como en el caso del montaje paralelo. Los tipos de montaje ruso tienen que ver con la extensión de los planos, con la sensación de ritmo y, desde la descripción que hace Eisenstein, se sugiere una mayor cercanía a conceptos musicales, como tono y duración. Ejemplos reconocibles de montaje estadounidense, desde Life of an American Fireman y de ahí en adelante hasta nuestros días. Los ejemplos reconocibles e inteligibles del montaje ruso ameritan una revisión más reflexiva. En el video Hardest Button to Button, del dúo White Stripes, dirigido por Michel Gondry (2003), es muy evidente el criterio de corte pensado para replicar la música que hay de fondo, y es un ejemplo preciso del montaje métrico de Eisenstein, y no necesariamente del montaje rítmico.

En este video de White Stripes, muchos de los fragmentos tienen como duración la que tiene cada golpe del bombo o bass drum, que a su vez coincide con cada pulso del bajo, y si la definición de Eisenstein hace referencia a "la extensión absoluta de los fragmentos", pues eso hace que este video musical sea un ejemplo preciso.

En cuanto al montaje rítmico, en el cual la sensación de ritmo es más importante que la duración absoluta de los fragmentos, en la introducción del documental Surplus, de Erick Gandini (2003), hay una música de fondo que guía los cortes sin que necesariamente determine la duración de los fragmentos. A partir del tiempo 00:02:15:00 se ve una imagen de un policía italiano que dispara una pistola de gases, y el sonido del disparo acompaña la acción. Cuatro segundos después, el disparo sigue sonando fuera de campo, y luego en intervalos de dos segundos. Del tiempo 00:02:35:00 en adelante, la música de fondo comienza a fundirse y solo queda el sonido ambiente de una protesta multitudinaria, pero el sonido del disparo de la pistola de gases sigue sonando a un intervalo de cada dos segundos, y el corte en adelante en ocasiones obedece a dicho intervalo, y en otras no.

Este es un buen ejemplo de montaje rítmico, en el que hay una sensación de ritmo clara, pero la duración de los fragmentos no necesaria ni recurrentemente es "métrica". Habría que hacer una distinción entre ambos ejemplos. En el video de Gondry, no se usa sonido ambiente para crear la sensación de ritmo, en el intro de Surplus sí se hace, y usa un sonido de disparo como pulso del ritmo. Siendo 
ambos ejemplos de los dos primeros hacer cine, para hacer video musical, tipos de montaje sugeridos por Ei- documental, como en Erik Gandini, senstein, ¿podría considerarse a esta o acaso algún otro tipo de creación forma de editar como método para video-musical?

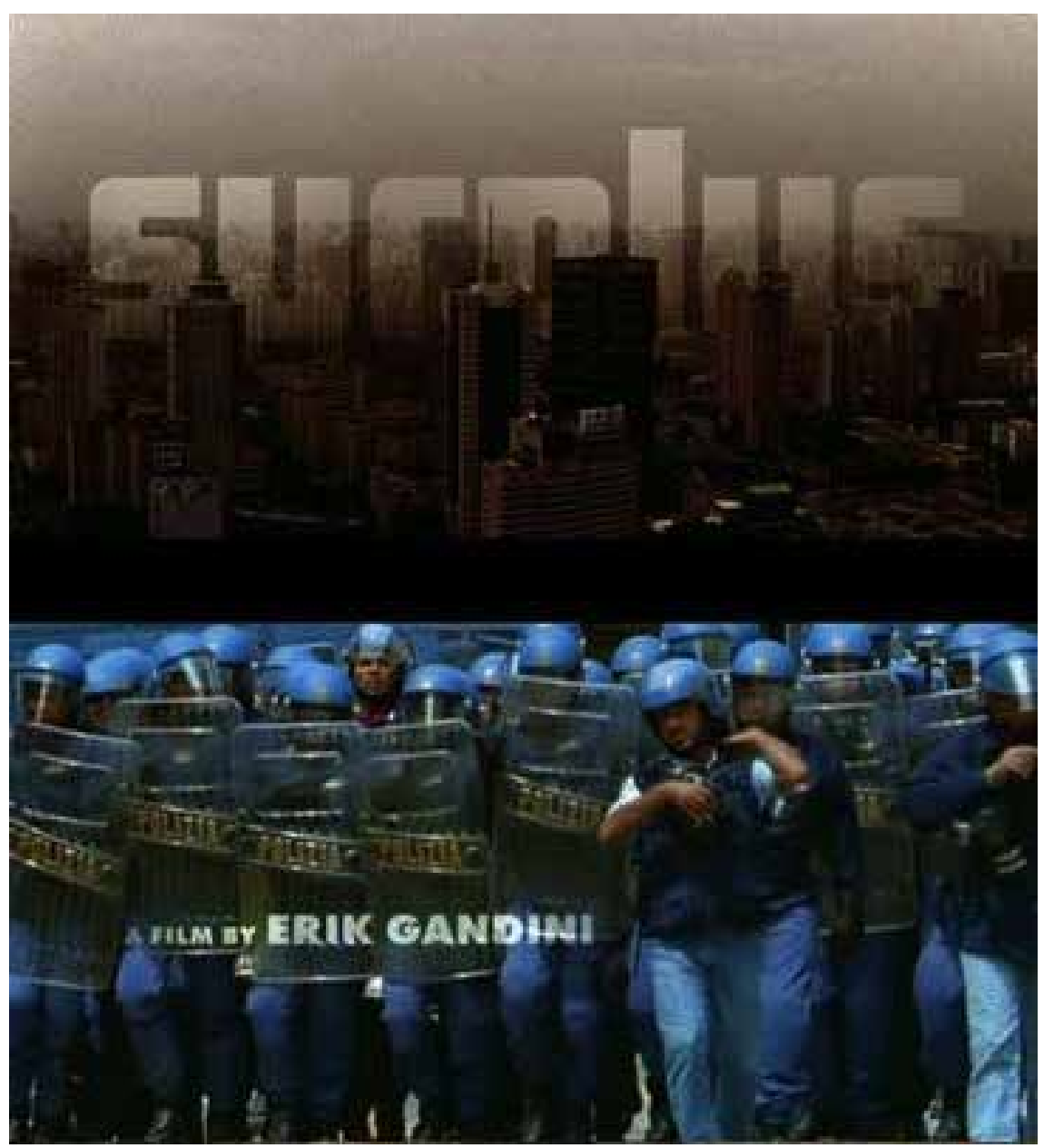

Erik Gandini, Surplus: Terrorized into being Consumers, 2003 (http://www.youtube.com/ watch? $\mathrm{v}=5 \mathrm{AE}$ EiwOM4fAY). 


\section{¿Es el videoloop realmente nuevo 0 es derivado de conceptos narrativos previos?}

En este artículo se quiere sugerir la introducción del término videoloop, definiéndolo como el producto de la manipulación de bucles o ciclos en el montaje de un video, que dan como resultado una musicalidad evidente tanto en lo sonoro como en el montaje de imágenes en movimiento. Desde los experimentos de Oskar Fischinger, o Fantasía, de Walt Disney, desde el cine se ha querido hacer una aproximación a la música y a sus conceptos, recreándola con imágenes mientras suena o tratando de parecérsele desde el montaje y la animación. El montaje métrico y rítmico, incluso el tonal y sobretonal, son herramientas conceptuales válidas para llevar a cabo dicho acercamiento; sin embargo, apreciar obras cinematográficas que hagan una valiosa aproximación a lo musical sacando provecho del montaje no es habitual. La música, en una película estándar, es un complemento emotivo del tono de una escena, marca un tema en toda una película con una melodía, acompaña las imágenes. Se antoja similar a lo hecho desde la ópera y la zarzuela siglos atrás, aunque ciertamente los musicales del cine, como los de Busby Berkeley en la década de 1930, eran mucho más cercanos a dichas puestas en escena orquestadas. De tal manera, la búsqueda de la musicalidad desde el ejercicio del montaje ha quedado en terrenos de lo experimental, alejado del mainstream, como en Oskar Fischinger.

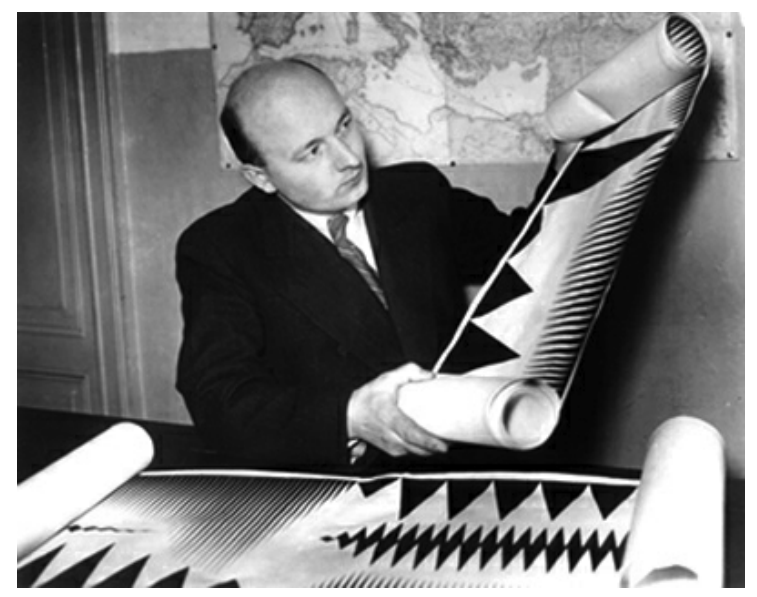

Oskar Fischinger (http://presented.se/blog/wp-content/upload/ ornament-soundjpg.jpeg). 


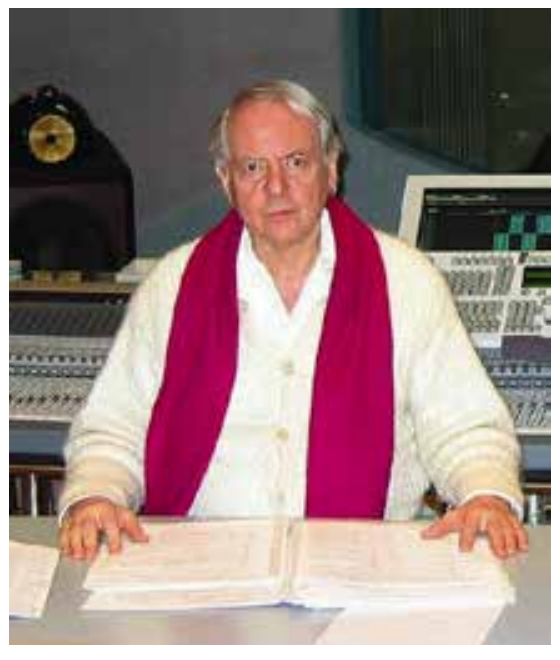

Karlheinz Stockhausen (http://commons.wikimedia. org/wiki/File:Stockhausen_March_2004_excerpt. ipg ?uselang=es)licencia creative commons).

Pero la búsqueda de la musicalidad desde el montaje no solo ha sido responsabilidad del cine mainstream o experimental. Desde lo musical, el compositor alemán Karlheinz Stockhausen ha hecho valiosos y valientes experimentos en música electroacústica, música aleatoria o para algunos tape music. En sus experimentos y admiradas creaciones, Stockhausen explora y lleva al límite los conceptos de tono, ritmo, duración, armonía y melodía. En ocasiones, pareciera que destruyera dichos conceptos por completo, pero al final sus obras se constituyen en una búsqueda por nuevos tonos, ritmos o armonías, y el carácter aleatorio de sus creaciones de alguna manera lo aleja del concepto de ritmo. Stockhausen hace una búsqueda musical pura.

En las décadas de 1970 y 1980, con el naciente uso de ordenadores personales para la creación multimedia, músicos y creadores audiovisuales integran tecnologías de la información y de la comunicación a sus flujos de trabajo, dando como resultado experimentos en lo musical y en lo audiovisual. El grupo aleman Einstürzende Neubauten en su video-musical Halber Mensch intenta crear música a partir de ruidos y unirlos con una creación musical a partir de bajo, guitarra eléctrica, batería y voz (http://www.youtube.com/watch?v=w2G3KfKbJwE).

Lo interesante de esta creación es el uso de ruidos capturados en video para alimentar la creación videomusical final. Ciertamente, desde la edición, sin un criterio métrico o rítmico, se logran compilar los mejores momentos de performance, pero se vuelve un primer intento en integrar video y ruidos en el montaje, con intenciones musicales.

En la música pop de la década de 1980, tomando la influencia de la música electrónica, se hacen loops o bucles de sonido ambiente y voces, intentando crear musicalidad desde la secuenciación de sonidos. En la canción Rock me Amadeus, Salieri Mix, 
de Falco (1985) se usan fragmentos de voces que dicen palabras como en una conversación, para apoyar y subrayar momentos de los pulsos de ritmo, y se reiteran para reforzar la sensación rítmica. Son voces que no están cantando entonadas, solo palabras sueltas que se repiten, logrando una clara musicalidad.

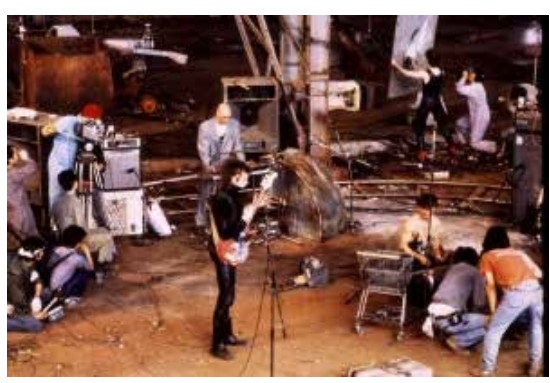

Einstürzende Neubauten, Halber mensch excerpt, 2006 (http://www.youtube.com/ watch? $\mathrm{v}=$ w2G3K FKbJwE Imagen obtenida en http://milinviernos.files.wordpress.com/2012/03/ einsturzende-neubauten-halber-mensch.jpg

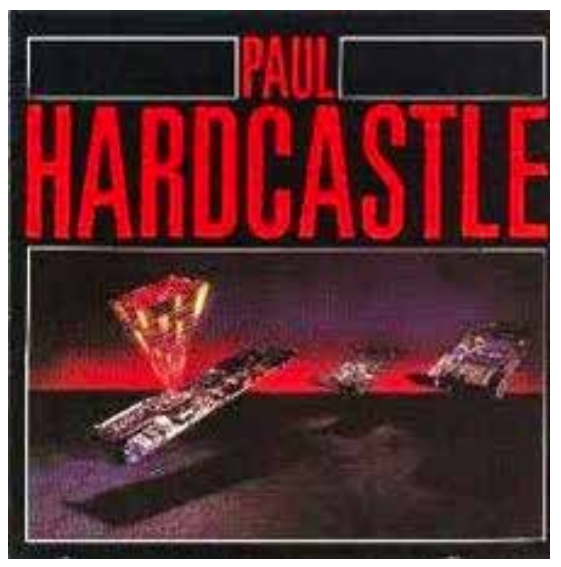

Paul Hardcastle, 19, 1985 (http://youtu.be/ oSGvqiVHik8 Fuente de la imagen: http:// 1 l.gstatic. com/images? q=tbn:ANd9GcTOI 1 L_h2uJE63U-P2 2N7AXWDSYKUW5t98d7k19_OrrkhapFuMbnpeT $\mathrm{dO} \mathrm{N}$ ).
En 19, de Paul Hardcastle, se usa un recurso similar a Rock me Amadeus, reiteración de voces, esta vez provenientes de un noticiero que habla sobre la guerra de Vietnam, repetidas en intervalos fijos para apoyar la percusión.

Se podría citar también el video y la canción de Pink Floyd Money, de 1973, con su loop de monedas y cajas registradoras, que se mezclan con el bajo inicial de Roger Waters, pero la pregunta es: ¿qué video de los ejemplos anteriores saca mejor provecho del material multiplexado capturado por la cámara? ¿Provecho que se saque tanto a la imagen y al sonido por igual sin supeditar imagen a sonido, o viceversa?

Asumir el reto de lograr musicalidad desde el ejercicio del montaje, reiterando momentos para crear ritmo, y escogiendo ciertos sonidos del material en video para que desde la reiteración adquieran cierto sentido, no está entre las intenciones estéticas de los creadores de los ejemplos anteriores. La música prácticamente se hace como un proceso aparte al de la edición, y la experimentación consiste en añadir y hacer bucle de ruidos y voces en una búsqueda del tono y del ritmo.

Buscar crear una creación musical solo con el video, solo con los 
ruidos capturados por una cámara de video, es el reto del videoloop. Comenzar en un proceso de creación audiovisual para terminar en un producto video-musical.

Hacer música ha sido desde siempre una vocación, un camino que demanda dedicación, estudio, actualización y reflexión. Por otra parte, hacer video, desde la aparición de aquella Sony Portapack a finales de la década de 1960, casi desde el principio fue sinónimo de rebeldía contra la televisión, curiosidad tecnológica, uso espontáneo de los recursos exclusivos de canales, empresa privada y Gobiernos. Desde las primeras obras de Nam June Paik y Wolf Vostell, hasta la convergencia entre cine y video de alta definición hoy, el video ha sido una suerte de plataforma tecnológica que a ratos se acerca a los modos de producción y productos televisivos, y a los modos y productos del cine, llegando a modos y productos propios en el videoarte $y$ el videoclip musical. En este último, la industria musical de finales de la década de 1970 y principios de la década de 1980, encontró en la tecnología del video la plataforma inicial para crear todo un movimiento social que finalmente promocionara comercialmente a los músicos y a sus trabajos discográficos en un mercado mundial con muchísimos rasgos, pero por efectos de la cultura dominante y sus ventajas históricas, técnicas y económicas, la industria del rock and roll y el pop anglo fue la más beneficiada en todo ese fenómeno sociocultural llamado videoclip musical. Pero en términos meramente formales, un video musical es la unión de formas narrativas cinematográficas, a veces estrictamente diegéticas, a veces experimentales, con una canción que en sí ya tenía un manejo formal propio de la música.

Gracias a los experimentos en video, en los que se juega con ruidos y reiteraciones, realizadores como Tanaka Hideyuki y Lasse Gjertsen, ambos disponibles en Youtube, podemos apreciar creaciones en video que sugieren una estructura musical. 


\section{Lasse Gjertsen}

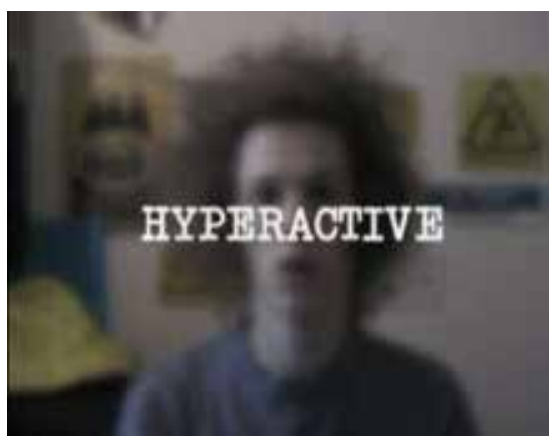

Lasse Gjertsen, Hyperactive, 2006 (http://www. youtube.com/watch? $\mathrm{v}=09698 \mathrm{Tq} \mathrm{Y}$ 4A).

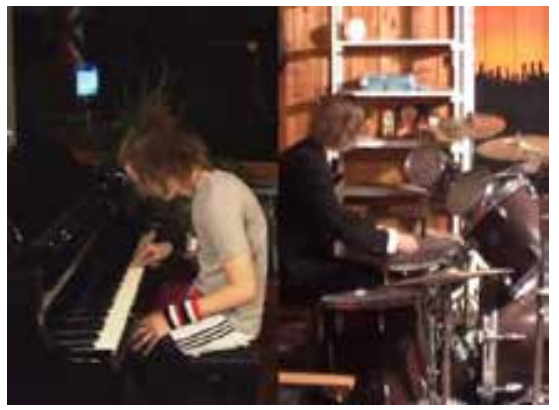

Lasse Gjertsen, Amateur, 2007 (http: / / www. youtube. $. \mathrm{com} /$ watch? $\mathrm{v}=\mathrm{J}$ zqumbhf $x$ Ro\&feature $=$ rela ted).

\section{Tanaka Hideyuki}

プリンストンガ (Princeton, la Polilla)

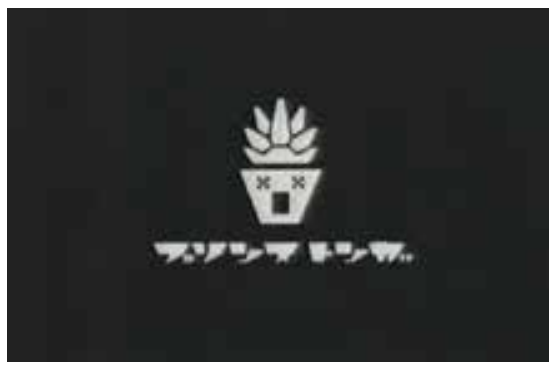

Tanaka Hideyuki, Bond TV, 2007 (http://youtu.be/ yBT4bgigyzE).
En Hyperactive y Amateur, Lasse Gjertsen usa sonidos de su boca o sonidos provenientes de instrumentos musicales para que, desde una edición precisa, se haga una creación musical usando video, es decir, usando material grabado desde una videocámara y, con el sonido capturado desde esta, se produce la ilusión sonora. Desde 2005, supe de los videos de un creador que figuraba en una compilación en DVD de Onedotzero identificado como Tanaka Hideyuki, y de inmediato me entusiasmé y me puse a hacer mis primeros intentos para Nokia Colombia, en un evento llamado Nokiatrends, que tuvo como evento principal un concierto de Moby. La agencia de publicidad (Grey and Partners o G2) del evento nos contrató al director Fernando López (http://vimeo.com/fernandolopez) y a mí para hacer piezas en video para el concierto y para diversos eventos, y de inmediato hice la propuesta de hacer un video como el de Tanaka Hideyuki. Fernando López, semanas antes, me había mostrado el DVD de Onedotzero (http://www.onedotzero.com/) en el que había muchos trabajos de artistas del video que mostraban una verdadera vanguardia en propuestas de montaje, narrativas y estéticas, entre ellos, Tanaka Hideyuki y un video 
llamado Bond TV (http://www.youtube.com/watch? $\mathrm{v}=\mathrm{Tj} 6 \mathrm{mzDMGBAY}$ ). El resultado de basar la propuesta para Nokia en lo que había hecho Hideyuki fue bautizado desde la agencia com Stomp, tal vez por la cercanía que tenía con el espectáculo estadounidense llamado Stomp Outloud (http://www.youtube.com/ watch?v=Zu15Ou-jKM0), que se podía apreciar en televisión por cable por esos días, y en el que personas en un escenario, con palos, baldes o tapas creaban virtuosas canciones, subrayando la precisión. La gran diferencia entre la creación musical de Stomp Outloud y la de Tanaka Hideyuki era precisamente la que diferencia teatro y cine: el montaje. En la primera, apreciamos una presentación en vivo; en la segunda, una creación cinematográfica que manipula espacio y tiempo con el corte. Lo que las vuelve similares es precisamente que son creaciones musicales sin instrumentos de música convencionales, haciendo una exploración con el timbre de los ruidos cotidianos, $y$ haciendo prácticamente caso omiso a cualquier escala tonal conocida.

\section{Felipe Cardona}

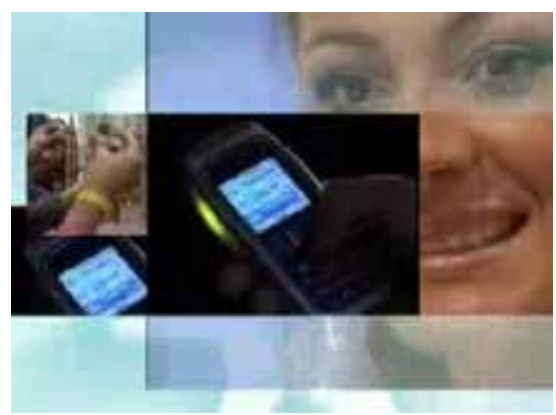

Felipe Cardona, Stomp, 2005 (http://youtu.be/ Bxps|XfiDxk).

Luego de terminar el encargo para Nokia y la agencia, llevé a cabo otros videos, usando varios de los recursos usados en Stomp. La técnica tenía mucho potencial, la exploración podía ser alrededor de diversos conceptos musicales. Inicialmente, se hizo alrededor del concepto de ritmo, pero había que buscar posibilidades alrededor del tono, el timbre, la melodía y la armonía. Los videos que le siguieron a Stomp fueron los siguientes:

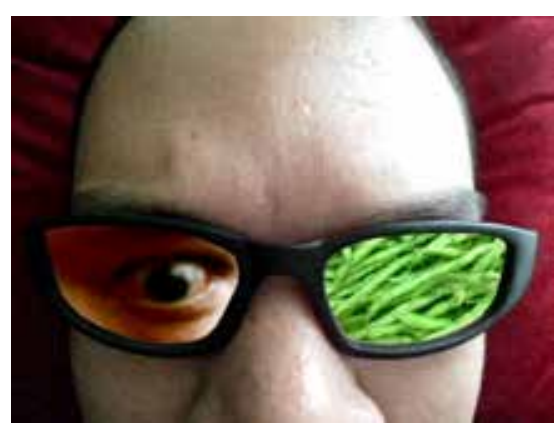

Felipe Cardona, Énfasis, 2007 (http://www youtube.com $/$ watch? $v=C 3 h \times r 7 \mid 7$ aill). 


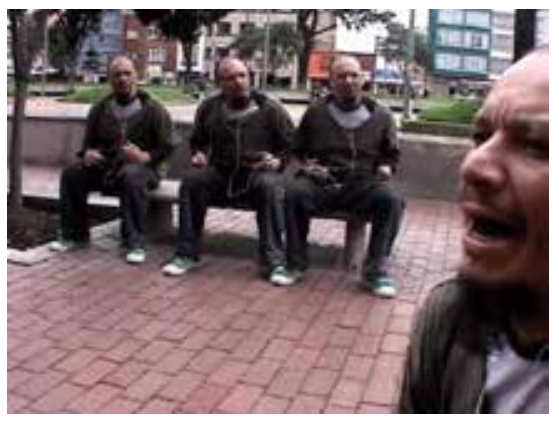

Felipe Cardona, El artista, 2007 (http://www. youtube. $. c 0 m /$ watch? $\left.v=f C k i \_E G d Q X M\right)$.

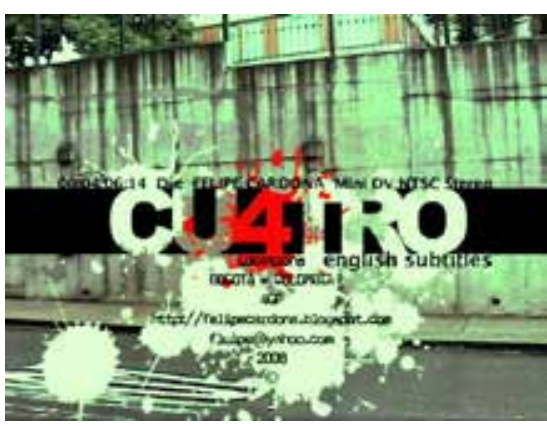

Felipe Cardona, Cu4tro, 2008 (http://www. youtube. $\mathrm{com} /$ watch? $v=$ MiXMB4s4kK8\&feature=re lated).

En Énfasis se pretende hacer una creación videomusical, grabada con un celular, en la que solo se usen ruidos y bucles del material grabado en video. Fue un gran reto, pero teniendo claridad sobre conceptos, como ritmo y duración, y buscando mínimas tonalidades (sonidos agudos, medianos y graves) fue suficiente para editar. En El artista se asumió el mismo reto de Énfasis, haciendo una creación más simple, usando los sonidos que un actor hacía frente a la cámara. En
Qu4tro se mezclan música y ruidos, en una única creación, usando bucles electrónicos de Drum'n Bass y ruidos como voces y ambiente, haciendo bucles de todo mezclado.

Es verdad que con mencionar la procedencia de ciertos recursos en la creación de los videos anteriores no queda del todo clara la metodología para su creación. Además, que la convergencia entre un flujo de trabajo en la edición de video y la creación musical amerita una descripción más detallada, para que quien se interese en hacer un videoloop tenga un punto de partida más certero.

\section{Cómo hacer música con video: hacia una metodología para crear un videoloop}

Entre 2010 y 2011 tuve la oportunidad de crear un taller de videoloop para una universidad y un festival de cortometrajes. El taller de videoloop que dicté en la Universidad de Manizales (Colombia) (http://www.umanizales. edu.co/) entre septiembre y octubre de 2011 y el taller en línea realizado para el festival HeARTbeat en Ekaterimburgo (Rusia) (http://www.youtube.com/watch?v=23stsYN8sSg) en abril de 2010 fueron prueba de cómo una posible metodología sobre el $v i$ deoloop podría lograr que alumnos 
inquietos sobre el tema resultaran al final con un producto videomusical interesante.

Los recursos formales que menciono en los videos anteriores, hablando específicamente de una exploración alrededor del concepto de ritmo, se resumen en dar una duración a cada fragmento editado en la línea de tiempo del programa de edición no lineal (Final Cut Pro, Premiere Pro), que tenga bastante similitud a la escala de duraciones que tienen las figuras musicales, desde la redonda (4 tiempos), la blanca (2 tiempos), la negra ( 1 tiempo), la corchea $(1 / 2$ de tiempo), la semicorchea (1/4 de tiempo), la fusa ( $1 / 8$ de tiempo), hasta la semifusa (1/16 de tiempo), haciendo equivalencias con duraciones de fragmentos de video en cuadros y segundos (asumiendo un segundo de NTSC como de 30 cuadros).

Hay otras figuras musicales de mayores duraciones (cuadrada, longa y máxima) y de tiempos más cortos que la semifusa (garrapatea y semigarrapatea), que ciertamente pueden también tener equivalencias en duraciones de video, siempre y cuando se tenga en cuenta que la menor duración en video NTSC y DVB-T es de 1 frame o cuadro, porque en un timeline no existe el 1/2 frame. Al final, las duraciones de una figura a otra se supeditan a multiplicar por dos o a dividir en dos la duración.

Esta equivalencia, en una línea de tiempo, podría ser la siguiente: 


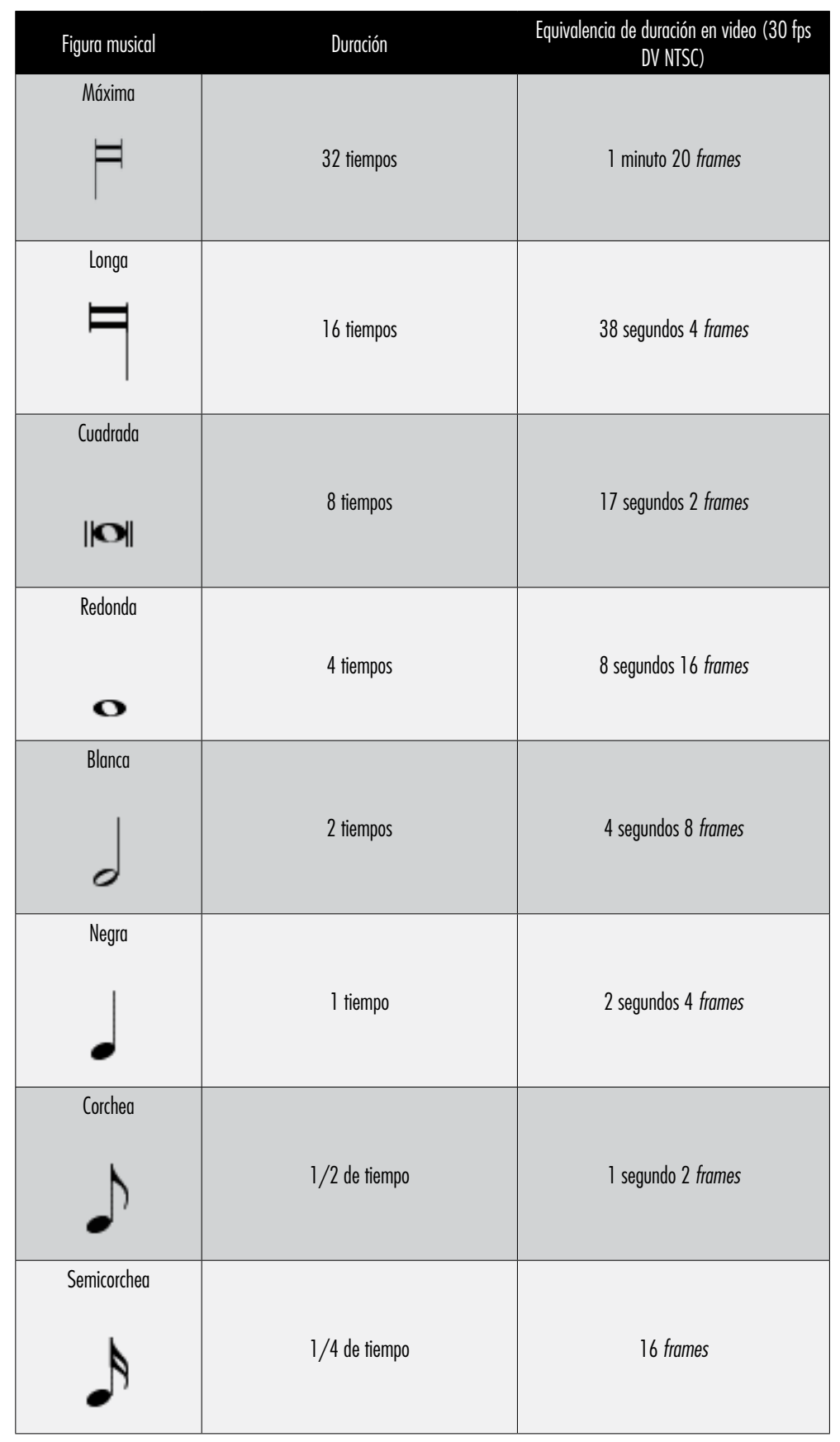




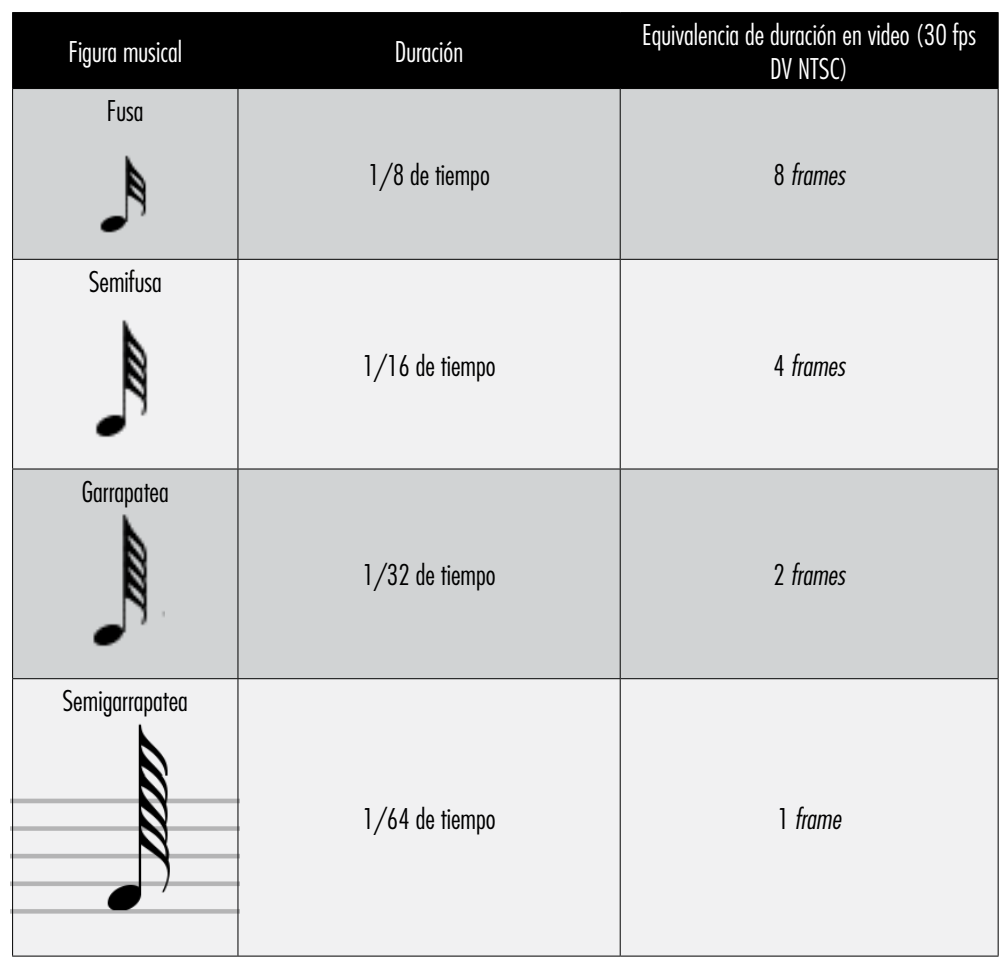

Figuras musicales tomadas de http://es.wikipedia.org/wiki/Figura_musical

Las duraciones son el todo en el ritmo, como se puede apreciar en una definición tras una búsqueda rápida en la web: "El ritmo se define como la organización en el tiempo de pulsos y acentos que perciben los oyentes en una estructura. Esta sucesión temporal se ordena en nuestra mente, percibiendo de este modo una forma" (http://es.wikipedia.org/wiki/Ritmo). También hay que entender que el ritmo, como una sucesión temporal de pulsos, no es exclusivo de la música, como lo señala la siguiente definición:
En un sentido general, el ritmo es un flujo de movimiento controlado o medido, sonoro o visual, generalmente producido por una ordenación de elementos diferentes del medio en cuestión. El ritmo es una característica básica de todas las artes, especialmente de la música, la poesía y la danza. También puede detectarse en los fenómenos naturales.

Existe ritmo en las infinitas actividades que gobiernan la existencia de todo ser vivo. Dichas actividades están muy relacionadas con los procesos 
rítmicos de los fenómenos geofísicos como las mareas oceánicas, el día solar, el mes lunar y los cambios de estaciones (http://es.wikipedia.org/wiki/ Ritmo).

El otro recurso es la búsqueda de cuatro tipos de sonido dentro de los ruidos que captura la cámara. Los sonidos buscados son:

- sonidos graves;

- sonidos medios;

- sonidos agudos;

- sonidos con timbre o tono variable (como una persona hablando).

Los sonidos graves se usan para simular el bass drum ('bombo') de una batería, los medios el snare drum ('redoblante') y los agudos los platillos, hi hat o crash. Los sonidos con timbre o tono variable los uso a modo de sampler y, editándolos de una manera reiterativa, se pueden equiparar, incluso, a una melodía o armonía, dependiendo de la edición y la reiteración. Lo importante de reiterar es hacerlo de manera orgánica, es decir, como la estructura musical de una canción. Lograr un buen loop o bucle, y reiterarlo solo dos veces, es un desperdicio de recursos. Reiterarlo hasta el final de la creación y sumarle nuevos bucles de diferentes duraciones que sean múltiplos o divisiones de la duración principal tiene ciertamente más posibilidad de enriquecer ese primer bucle.

De esta manera, la producción en video se acerca a lo que nunca se había acercado apropiadamente, a la construcción musical. Antes se usaba la música para complementar al video, o al video para promocionar la música de algún artista. En este caso particular, se hace una creación en video que también es creación musical.

\section{Conclusiones}

Cuando Eisenstein describe los métodos de montaje rítmico y métrico, habla de duraciones y de ritmo. El criterio de clasificación de dichos métodos provenía de terrenos musicales, diferente al cine estadounidense, cuyos métodos de montaje son provenientes de la literatura. Cuando aparece el cine sonoro, y cuando Eisenstein, Alexandrov y Pudovkin abogan por un uso del sonido innovador desde su Manifiesto del contrapunto sonoro, buscaban desde ese momento que futuros cineastas reflexionaran sobre el montaje, y lo llevaran al límite de sus posibilidades, ampliadas a partir del sonido. Con la aparición de las cámaras de video 
para usuarios finales, a partir de la Portapack de Sony, video y audio vienen unidos en una única señal, lo que ha hecho que los usuarios lleguen a pensar que el acompañamiento de sonido e imagen sea natural. Nada más ilusorio y perjudicial para las intenciones de aquel Manifiesto del contrapunto sonoro.

Hoy, cuando Youtube le permite a cualquier usuario subir y exhibir sus videos al mundo, la posibilidad de que usuarios no iniciados en el cine ni en la televisión se atrevan a probar y llevar al límite el montaje ha hecho que surjan creaciones en video, como las de Lasse Gjertsen, que refrescan el panorama de uso que los estadounidenses le han dado al montaje, y los límites que su industria mainstream internacional le ha puesto a la innovación. Viendo estos nuevos videoloops, vuelven a la vida aquellos conceptos de montaje de los cineastas rusos de la década de 1920, y sus ganas de ir siempre un poco más allá de las posibilidades del montaje. Se materializan teorías, en creaciones que retan los usos del montaje de la ficción, incluso del videoclip musical. Con reiteraciones simples que se complejizan al antojo del creador, se crean ilusiones de sentido, sensaciones musicales con tonos provenientes de ruidos, voces $y$, en general, todo tipo de sonido ambiente. Ruido que se vuelve música, montaje cinematográfico que le sirve a la composición. Nueva tecnología y democratización de flujos de trabajo que les permiten a usuarios finales, hoy prosumidores, enseñarles a canales de televisión, estudios de cine e, incluso, casas disqueras mainstream, cuál puede ser el rumbo futuro de la música, el cine y la televisión.

\section{Referencias bibliográficas}

Beardbox (2009). Recuperado de http://www.youtube.com/watch?v= CHMbCodWCnU\&feature=youtu.be

Berchenko Acevedo, S. (2012). Figuras simples. Recuperado de http:// web.mac.com/sberchenko/Notacion_ Musical/Figuras_simples.html

Butler, J. (2007/1990). El género en disputa: el feminismo y la subversión de la identidad. Barcelona: Paidós.

Cardona, F. (2006). Stomp. Recuperado de http://www.youtube.com/watc $\mathrm{h} ? \mathrm{v}=\mathrm{BxpsJXfiDxk \& feature=youtu.be}$

Cardona, F. (2008). El artista. Recuperado de http://www.youtube .com/watch?v=fCki_EGdQXM\& feature=youtu.be 
Cardona, F. (2008). CU4TRO. Recuperado de http://www.youtube.com/ watch? $\mathrm{v}=\mathrm{MiXMB} 4 \mathrm{~s} 4 \mathrm{kK} 8 \&$ feature $=\mathrm{y}$ outu.be

Cardona, F. (2010). Énfasis. Recuperado de http://www.youtube.com/ watch?v=C3hxr7I7aiI

Chion, M. (2004). La voz en el cine. Madrid: Cátedra.

Einstürzende Neubauten (2012). Recuperado de http://es.wikipedia. org/wiki/Einst\%C3\%BCrzende_Neubauten

Einstürzende Neubauten-halber mensch excerpt (2006). Recuperado de http://www.youtube.com/ watch?v=w2G3KfKbJwE

Eisenstein, S. M. y Gubern, R. (eds.) (1970). Reflexiones de un cineasta. Recuperado de http:// en.scientificcommons.org/6876771

Eisenstein, S. M. (1986). La forma del cine. México: Siglo XXI.

Eisenstein, S. M. (1997). El sentido del cine. México: Siglo XXI.
Fernández, M. (2008). Pensar el cine. Un repaso histórico a las teorías cinematográficas. Madrid: Lecciones del portal. Recuperado de http://www. portalcomunicacion.com

Figura musical (2012). Recuperado de rom http://es.wikipedia.org/wiki/ Figura_musical

Funny little girl beatbox (riezinha159) (2008). Recuperado de http://www. youtube.com/watch?v=ASucb7x1Gl $\mathrm{k} \&$ feature=youtu.be

Gjertsen, L. (2006). Amateur. Recuperado de http://www.youtube.com/ watch? $v=$ JzqumbhfxRo\&feature $=y$ outu.be

Gjertsen, L. (2006). Hyperactive-lasse gjertsen. Recuperado de http://www. youtube.com/watch?v=09698TqtY4A $\&$ feature=youtu.be

Hideyuki, T. (2007). Bond TV

プリンストンガ Recuperado de http:// www.youtube.com/watch?v=yBT4bg jgyzE\&feature=youtu.be

Karlheinz stockhausen. (2012). Recuperado de http://en.wikipedia.org/ wiki/Karlheinz_Stockhausen 
Kracauer, S. (1989). Teoría del cine. La zenelectrónico: Nam June Pai. Ars redención de la realidad física. Barce- Longa, 18, 173-183.

lona: Paidós.

Stam, R. (2010). Teorías del cine. Una

Ferla, J. la (2009). Cine (y) digital: introducción. Madrid: Paidós.

aproximaciones a posibles convergen-

cias entre el cinematógrafo y la com-

Stockhausen: Telemusik (2011). Re-

putadora. Buenos Aires: Manantial.

cuperado de http://www.youtube. com/watch?v=jcwLDxlNKdU\&feat

Pudovkin, V. I. (1957). Lecciones de ure=related cinematografía. Madrid: Rialp.

Sarriugarte Gómez, Í. (2009). El inefable sendero del budismo 